\title{
Transient thermal resistance of borehole heat exchangers for hourly simulations of geothermal heat pumps systems
}

\section{Antonella Priarone}

\author{
Marco Fossa
}

\begin{abstract}
The correct design of borehole fields requires the correct evaluation of the transient ground thermal response in time, but also the accurate estimation of the borehole (BHE) thermal resistance, expecially the grout contribution. Generally, the borehole thermal resistance is considered as steady-state; however, when considering the borefield hourly response to the building variable thermal loads, also the transient behavior of the grout thermal resistance plays an important role, which is quite often neglected. This study analyzes, with a dimensionless approach, the transient grout thermal resistance, with particular attention devoted to the effect of the boundary condition imposed to the internal tubes, namely imposed heat flux, imposed temperature and imposed convective coefficient, the last being the real operating conditions. In addition, the effects of grout to ground thermophysical properties and of shank spacing are analysed. The steady state numerical results are also compared with literature correlations. Finally, numerical evidences are given to demonstrate that the usual approach of calculating the overall BHE resistance just summing the grout resistance, numerical obtained by imposing a temperature on the tube surface, to the convective one can lead to meaningful errors at low Biot numbers.
\end{abstract}

\section{INTRODUCTION}

Ground Coupled Heat Pumps (GCHPs) are a high efficiency solution for building conditioning in the framework of energy saving and environmental protection. In most applications the heat pump is coupled with the ground by means of Borehole Heat Exchangers (BHEs) and it takes advantage of the favourable ground temperatures for high efficiency heating and in cooling heat transfers. The most complete way for analysing the thermal behaviour of the GCHP system as a whole is an hourly approach, able to forecast and optimize the system response to variable thermal loads.

This approach is quite different from the methods commonly used to size and examine the operating behaviour of the BHE field, since the typical approach is to consider the ground response to simplified heat load profiles, typically with a monthly time step. In this framework, it is a common practice to use a two resistances scheme to model the thermal behaviour of a BHE field. In particular, the first resistance depicts the thermal response of the ground, intrinsically variable in time, and is commonly described by proper Temperature Response Factors (TRF), also known as $g$-functions. On the contrary, the second resistance represents the BHE thermal behaviour (Yavuzturk and Spitler 1999; Zeng, et al. 2003; Marcotte and Pasquier 2008) and is frequently considered constant in time. Lamarche et al. (2010) presented a wide review of methods and correlations to evaluate the borehole thermal resistance, focusing in particular on the grout thermal resistance. More recently Javed and Spitler (2016) compared a 
wide range of models for calculating borehole thermal resistance and recommended suitable methods also for water filled boreholes.

The aim of this work is to analyze the transient behavior of the grout thermal resistance by means of numerical simulations performed in COMSOL Multiphysics environment. The modelled domain is a $2 \mathrm{D}$ cross section of the BHE and the modelling is performed by introducing proper dimensionless quantities. Different aspects are considered, including the effects of the thermal conductivity and heat capacity values, in terms of grout to ground ratio: the dimensionless grout thermal resistance appears to be a function of both these ratios but reaches a constant steady-state value for specific threshold values of the radius based Fourier number.

A special attention has been devoted to the investigation of the influence of the boundary condition applied on the pipes side of the BHE: imposed heat flux, imposed temperature and imposed convective coefficient. For a U-pipe BHE, due to its non-axialsymmetrical geometry, the choice of the proper boundary conditions is revealed to be important, because it significantly influences not only the transient trend of the BHE thermal resistance but also its steady state value.

\section{THEORETICAL BACKGROUND}

The borehole thermal resistance $R_{b}$ includes different contributions, namely the convective resistance of the fluid, the conductive resistance of the pipes and the conductive resistance of the grout. The first two resistances can be easily calculated, whereas the evaluation of the third requests analytical correlations or numerical simulations (Figure 1). In this paper the analysis is devoted exclusively to the grout thermal resistance $R_{g t}$, which is typically the main contribution; the usual approach to obtain the effective borehole thermal resistance $R_{b}$ is to add the convective and pipe contributions $R_{p p}$ to $R_{g t}$ (Eq. 1 and 2, single $U$ case), even if this can result in meaningful errors, as discussed later in this paper.

$$
\begin{aligned}
& R_{b}=R_{g t}+R_{f p} / 2 \\
& R_{f p}=\frac{1}{2 \pi}\left(\frac{\ln \left(r_{p, \text { out }} / r_{p, \text { in }}\right)}{k_{p}}+\frac{1}{r_{p, \text { in }} \cdot h}\right)
\end{aligned}
$$

In the following, the main correlations to evaluate the grout thermal resistance are presented. Considering the borehole radius $r_{b}$, the external pipes radius $r_{p}$, the half shank spacing $d$, the ground volume radius $r_{g}$, and the grout and ground thermal conductivities $k_{g t}$ and $k_{g}$, respectively, the following dimensionless variables are introduced:

$$
d^{*}=\frac{d}{r_{b}} \quad r_{p}^{*}=\frac{r_{p}}{r_{b}} \quad r_{g}^{*}=\frac{r_{g}}{r_{b}}
$$
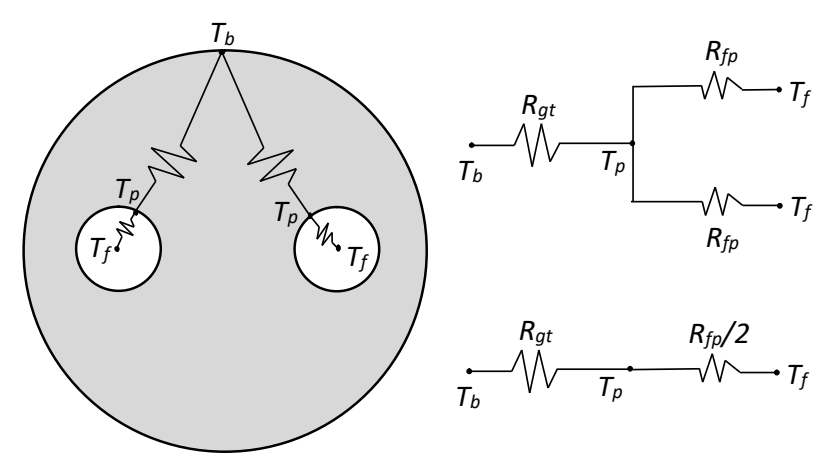

Figure 1 Sketches of the thermal resistances. 


$$
\begin{aligned}
& k^{*}=\frac{k_{g t}}{k_{g}} \quad \sigma=\frac{k^{*}-1}{k^{*}+1} \\
& R_{g t}^{*}=\left(2 \pi \cdot k_{g t}\right) \cdot R_{g t}
\end{aligned}
$$

Paul (1996) proposed a very simple correlation, in which the dimensionless grout thermal resistance $R_{g t}{ }^{*}$ depends on the ratio of pipe to borehole radius $r_{p}^{*}$, and on the half shank spacing between the pipes $d\left(\beta_{0}\right.$ and $\beta_{1}$ are constants, whose values depend on the distance $d$, see Figure 2):

$$
R_{g t}^{*}=\frac{2 \pi}{\beta_{0}\left(1 / r_{p}^{*}\right)^{\beta_{1}}}
$$

Hellstrom (1991) proposed the so called 'line source formula', depending also on the ratio of grout to ground thermal conductivities $k^{*}$, by means of the parameter $\sigma$.

$$
R_{g t}{ }^{*}=\frac{1}{2} \cdot\left[\ln \left(\frac{1}{r_{p}^{*}}\right)+\ln \left(\frac{1}{2 \cdot d^{*}}\right)+\sigma \cdot \ln \left(\frac{\left(1 / d^{*}\right)^{4}}{\left(1 / d^{*}\right)^{4}-1}\right)\right]
$$

Bennet et al. (1987) proposed a complex algorithm, called 'multipole method' for calculating the overall thermal resistance $\mathrm{R}_{b}$. Based on Eq. 1 formulation, the Bennet expression (first-order approximation) can be rearranged as:

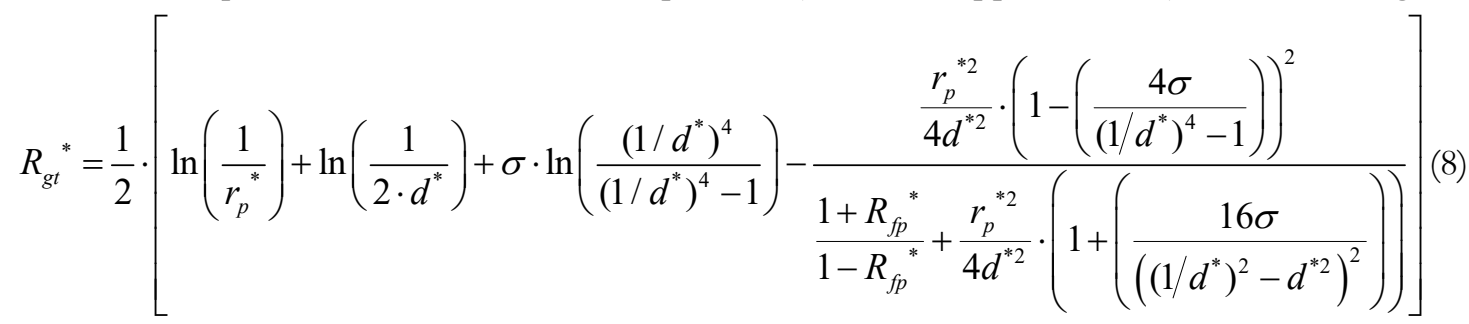

Neglecting the pipe contribution, the dimensionless convective resistance can be expressed as:

$$
R_{f p}{ }^{*}=2 \pi \cdot k_{g t} \cdot R_{f p} \cong 2 \pi \cdot k_{g t} \frac{1}{2 \pi \cdot r_{p} \cdot h}=\frac{1}{B i \cdot r_{p}^{*}}
$$

where the Biot number is here defined as $B i=h \cdot r_{b} / k_{g t}$.

Finally, Sharqawy et al. (2009) more recently suggested the following simple correlation:

$$
R_{g t}{ }^{*}=\left[-1.49 \cdot d^{*}+0.656 \cdot \ln \left(\frac{1}{r_{p}^{*}}\right)+0.436\right]
$$

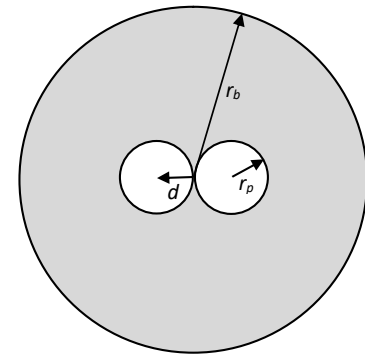

Case A: $d^{*}=0.289$ $\beta_{0}=20.10 ; \beta_{1}=-0.9447$

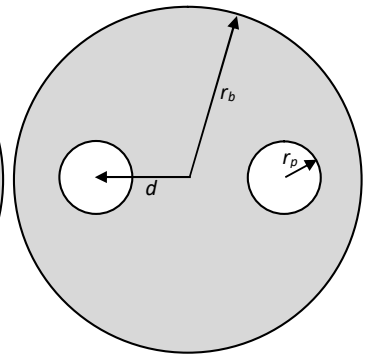

Case B: $d^{*}=0.367 ; 0.5$ $\beta_{0}=14.44 ; \beta_{1}=-0.605$

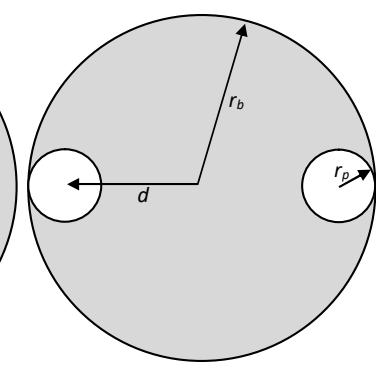

Case C: $d^{*}=0.695$ $\beta_{0}=21.91 ; \beta_{1}=-0.3796$

Figure 2 Present paper test cases for single U pipe heat exchanger. 
These correlations are based on the assumption that the borehole thermal resistance can be determined for quasi-steady state conditions and depends mainly on geometrical parameters and on grout and ground thermal conductivities. On the contrary, the first order approximation of the Bennet et al. correlation takes into account, for the evaluation of the grout thermal resistance, also the convective resistance contribution that influences the temperature field in the grout domain.

\section{GOVERNING EQUATIONS AND NUMERICAL MODEL}

In this paper single U-pipe BHE is considered, with $r_{b}=7.62 \mathrm{~cm}$ and $r_{p}=2.1 \mathrm{~cm}\left(r_{p}^{*}=0.275\right)$ (based on commercially available geothermal probes). Four different shank spacing values are considered, namely $d^{*}=0.289$; $0.367 ; 0.5 ; 0.695$; the first value leads to a configuration like case $\mathrm{A}$ of Figure 1 , the second and third values to a configuration like case $\mathrm{B}$, and the last one to a configuration like case $\mathrm{C}$.

The model analyzes a $2 \mathrm{D}$ cross section of the BHE and the considered domains are the grout and an appropriate surrounding portion of ground. The grout thermal resistance is assumed to be time dependent; thus, the differential equations in the grout and the ground are, respectively:

$$
\begin{aligned}
& \frac{\partial T}{\partial \tau}=\frac{k_{g t}}{(\rho c)_{g t}} \nabla^{2} T \\
& \frac{\partial T}{\partial \tau}=\frac{k_{g}}{(\rho c)_{g}} \nabla^{2} T
\end{aligned}
$$

Three different boundary conditions have been imposed at the surface between pipes and grout, namely imposed heat flux per unit length $\dot{Q}^{\prime}$, imposed temperature $T_{p}$ and convective heat transfer with imposed fluid temperature $T_{f}$ and convective coefficient $h$ :

$$
\begin{aligned}
& -\left.k_{g t}(\nabla T \cdot \mathbf{n})\right|_{S}=\frac{\dot{Q}^{\prime}}{4 \pi \cdot r_{p}} \\
& T=T_{p} \\
& -\left.k_{g t}(\nabla T \cdot \mathbf{n})\right|_{S}=h\left(T-T_{f}\right)
\end{aligned}
$$

The external boundary of the computational domain, which is a circle with radius $r_{g}$, is considered isothermal

$$
T=T_{g r, \infty}
$$

where $T_{g r, \infty}$ is the undisturbed ground temperature.

Continuity conditions hold at the interface grout to ground, and the initial condition is $T=T_{g, r}$ on the whole computational domain.

The study and the equation solution are developed in a dimensionless form, introducing different dimensionless variables, depending on the boundary conditions. Thus, the following dimensionless time $\tau^{*}$ and temperatures $T^{*}$ are defined:

$$
\tau^{*}=\frac{k_{g} \cdot \tau}{(\rho c)_{g} r_{b}^{2}}=F o_{r b}
$$

$$
\begin{array}{ll}
\text { Imposed heat flux, } & T^{*}=k_{g} \frac{T-T_{g r, \infty}}{\dot{Q}^{\prime}} \\
\text { Imposed temperature, } & T^{*}=\frac{T-T_{g r, \infty}}{T_{p}-T_{g r, \infty}}
\end{array}
$$


Imposed convection,

$$
T^{*}=\frac{T-T_{g r, \infty}}{T_{f}-T_{g r, \infty}}
$$

By introducing also the dimensionless quantity: $\quad(\rho c)^{*}=\frac{(\rho c)_{g t}}{(\rho c)_{g}}$

it is possible to rewrite Eqs. (11), (12), (14) in the following dimensionless form:

$$
\begin{aligned}
& \frac{\partial T^{*}}{\partial \tau^{*}}=\frac{k^{*}}{(\rho c)^{*}} \nabla^{* 2} T^{*} \\
& \frac{\partial T^{*}}{\partial \tau^{*}}=\nabla^{* 2} T^{*} \\
& T^{*}=0
\end{aligned}
$$

The dimensionless initial condition is $T^{*}=0$ everywhere in the domain.

The different boundary conditions become:

$$
\begin{aligned}
& -\left.\left(\nabla^{*} T^{*} \cdot \mathbf{n}\right)\right|_{S^{*}}=\frac{1}{4 \pi k^{*} r_{p}^{*}} \\
& T^{*}=1 \\
& -\left.\left(\nabla^{*} T^{*} \cdot \mathbf{n}\right)\right|_{S}=B i \cdot\left(T^{*}-1\right)
\end{aligned}
$$

The analysis of the effects of the convective coefficient is carried out for $B i$ values equal to 10 and 50.

In order evaluate the effect of the thermophysical properties of grout and ground, different combinations of the following values have been considered: $k^{*}=0.3 ; 0.6 ; 1.0 ; 1.667 ;(\varrho c)^{*}=0.4 ; 0.7 ; 1.0$.

The dimensionless Eqs. (18)-(20), with the continuity conditions at the interface grout-ground, the initial condition and the boundary conditions (21) have been solved by means of COMSOL Multiphysics.

A dimensionless time interval $10^{-4} \leq \tau^{*} \leq 10^{5}$ is considered, which is divided into 4500 uniform time steps (each dimensionless time interval is equal to 0.002).

A circular computational domain representing the ground volume is employed, with dimensionless radius $r_{g}^{*}=1000$; an extensive check of the adequacy of this size of the computational domain has been already performed by Zanchini and Lazzari (2014), to which the present work refers.

For the analysis of the mesh suitability, the criteria here adopted follows the work by Priarone and Lazzari (2014), even if in the present investigation the elements number is further increased for a more accurate calculation of temperatures and heat fluxes on pipes and BHE boundaries. Finally, an unstructured triangular mesh is chosen, which presents 200 uniformly spaced elements on each pipe boundary and 400 elements on BHE boundary, for an overall number of elements equal to about 24000.

\section{RESULTS AND DISCUSSION}

The transient behaviour of the dimensionless grout thermal resistance $R_{g t}{ }^{*}$ is presented in Figures 3 and 4 for an intermediate value of the shank spacing $\left(d^{*}=0.5\right)$.

In particular, Figure 3 allows the effect of the boundary conditions to be appreciated: the trends are different for both the transient zone and the steady state values, with a different $F_{o_{r b}}$ value at which they reach the asymptote. The grout thermal resistance for imposed heat flux is lower for the small values of $F_{0 r b}$ whereas it reaches a considerably higher value in the steady state zone. The trends of $R_{g t}^{*}$ for the imposed temperature and convective boundary conditions seem to have a comparable behaviour, more similar for higher value of Biot number: in fact, for $B i=50$ (corresponding approximately to a convective coefficient $h=2000 \mathrm{~W} / \mathrm{m}^{2} \mathrm{~K}$ ) the two curves nearly overlap. 
On the contrary, Figure 4 shows the effect of the dimensionless heat capacity for unit volume $(\rho c)^{*}$ on the transient behaviour of the dimensionless grout thermal resistance $\mathrm{R}_{g t}{ }^{*}$ : increasing the $(\rho c)^{*}$ value the grout thermal resistance is decreased for all the analysed boundary conditions here taken into account. Conversely, the dimensionless heat capacity $(\rho c)^{*}$ does not affect the steady value of the dimensionless grout thermal resistance $R_{g t}{ }^{*}$, as expected from the steady formulation of the conduction equations.

The steady values of $R_{g t}{ }^{*}$ calculated by means of correlations $(7,8,10)$ are reported in both Figures 3 and 4: Paul correlation provides a value of 0.95 , much higher than all the numerical results and it is outside of the selected axis range. A very good agreement exists between numerical results and Bennet et al. estimations (Eq. 8) when in present simulations the convection boundary condition is applied. The value of $R_{g t}{ }^{*}$ from Hellstrom correlation is intermediate between the imposed temperature and imposed heat flux numerical values, whereas the Sharqawy value is considerably lower than all the numerical results.

Figure 5 compares the steady state values of $R_{g t}^{*}$ obtained by means of numerical simulations with those from literature correlations. In particular, Figure 5(a) analyses the effect of the dimensionless thermal conductivity $k^{*}$, showing a small increase of $R_{g t}{ }^{*}$ with it. On the contrary, in Figure $5(b)$ the dimensionless grout thermal resistance $R_{g t}{ }^{*}$ decreases by increasing the dimensionless shank spacing $d^{*}$.

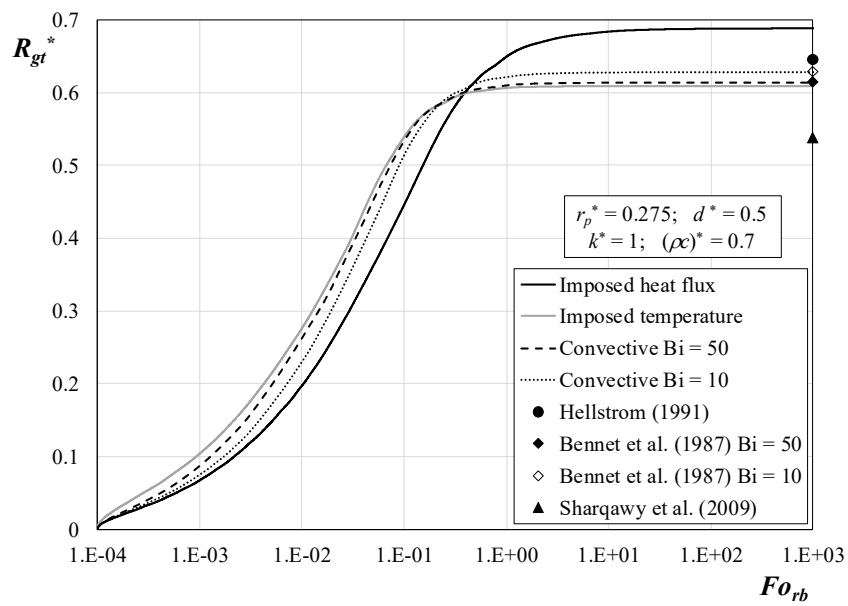

Figure 3 Transient behaviour of the dimensionless grout thermal resistance $R_{g t}{ }^{*}$ for the three boundary conditions and for $d^{*}=0.5, k^{*}=1,(\rho c)^{*}=0.7$. Comparison with literature correlation steady values $\left(R_{g t}^{*}\right.$ Paul $\left.=0.95\right)$.

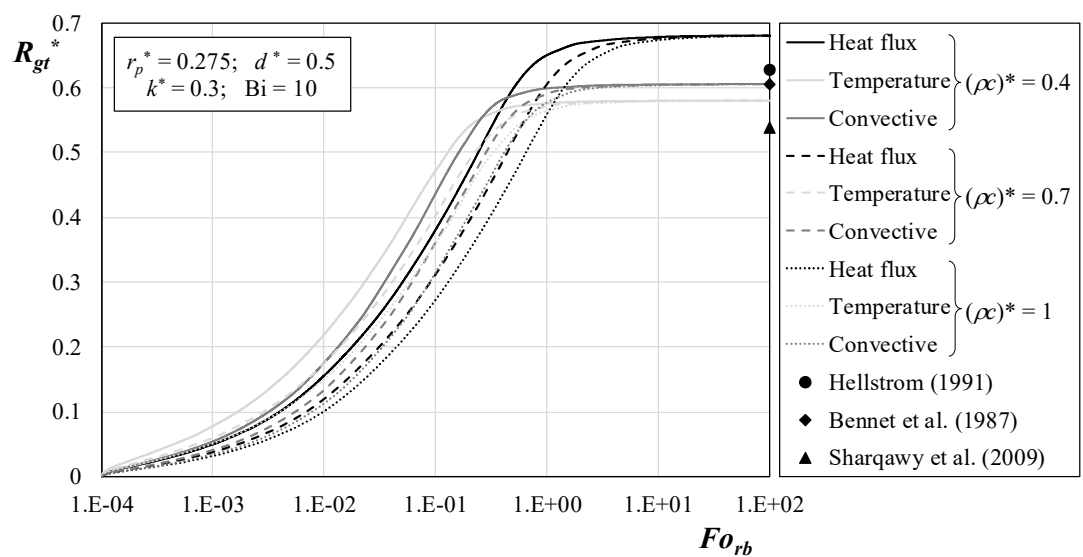

Figure 4 Effect of the dimensionless heat capacity $(\rho c)^{*}$ on the transient behaviour of the dimensionless grout thermal resistance $R_{g t}{ }^{*}$ for the three boundary conditions for $d^{*}=0.5, k^{*}=0.3, B i=10$. Comparison with the literature correlation steady values (Paul 1996 value is out of the axis range: $R_{g t}{ }^{*}$ Paul $=0.95$ ). 


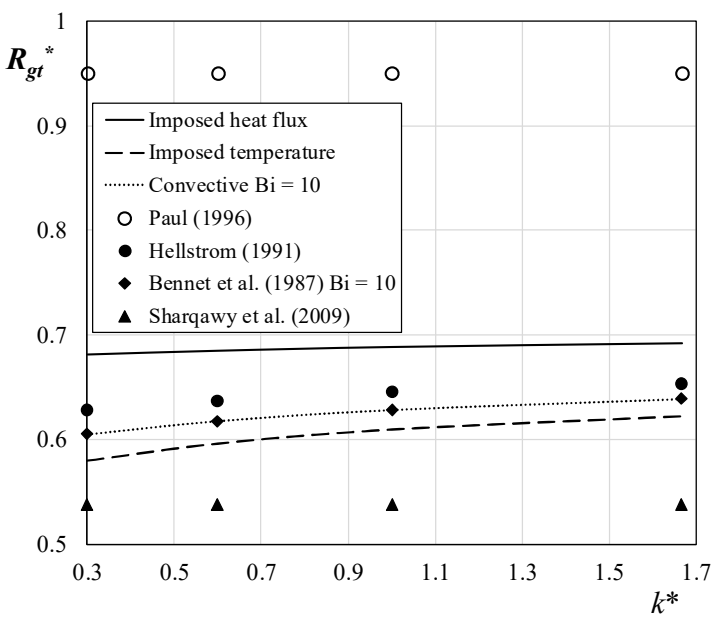

(a)

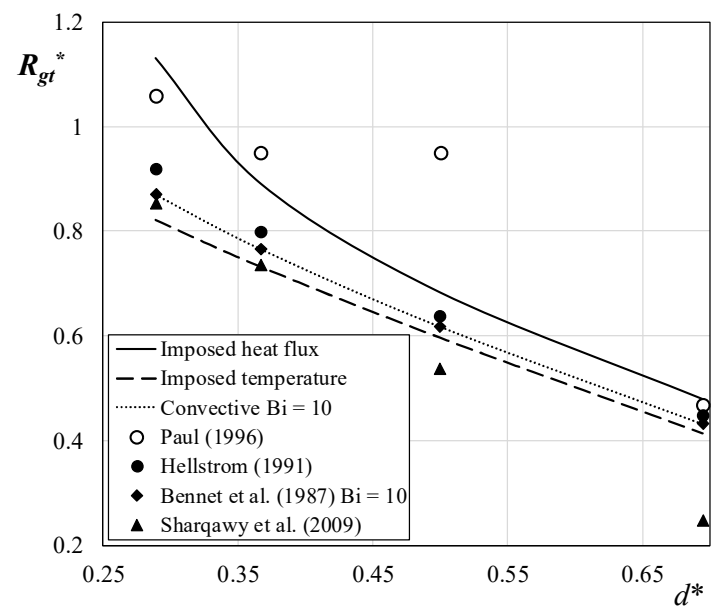

(b)

Figure 5 Steady state values of the dimensionless grout thermal resistance $R_{g t}{ }^{*}$ : (a) Influence of the dimensionless thermal conductivity $k^{*}\left(d^{*}=0.5, B i=10\right)$; (b) Influence of the dimensionless shank spacing $d^{*}\left(k^{*}=0.6, B i=10\right)$.

One of the most interesting results of this analysis is the different values of the dimensionless grout thermal resistance $R_{g t}{ }^{*}$ obtained by applying different boundary conditions on pipe side, namely imposed temperature or imposed convective coefficient. In fact, the non-assialsymmetry of the geometry leads to different temperature fields in the grout, as already stressed by a previous paper of this research group (Fossa and Dalla Pietà, 2011).

Thus, the usual approach of simply adding the convective thermal resistance (often neglecting the pipe's one) to the grout thermal resistance obtained by imposing a temperature boundary condition, is revealed to be uncorrect and leading to significant errors. On the other hand the comprehensive approach by Bennet et al. is able to efficiently describe the heat transfer characteristics when a convective boundary condition is applied.

Figure 6 presents the results for a double U pipe, comparing the dimensionless grout plus convective thermal resistances $R_{g t+f}^{*}$ calculated with two different approaches. The value obtained by applying the imposed temperature boundary condition and then adding the convective thermal resistance significantly understimates the value obtained by directly applying the convective boundary condition, with an error that increases for low values of the Biot number. In particular, the average percentage relative error at the steady state asymptote is $\varepsilon=3.4 \%$ for $B i=50$ and $\varepsilon=10.7 \%$ for $B i=10$.

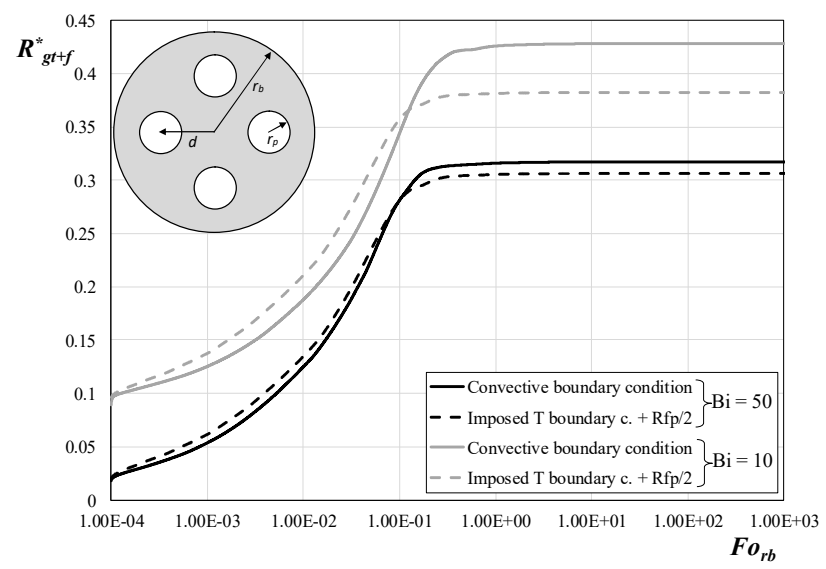

Figure 6 Effect of different boundary conditions on the dimensionless grout plus fluid thermal resistance $R_{g t+f}^{*}\left(r_{t}^{*}=0.263 ; d^{*}=0.592 ; k^{*}=0.6\right)$ 


\section{CONCLUSIONS}

In this paper the grout thermal resistance of a $\mathrm{U}$ pipe borehole is analysed by means of numerical simulations, by applying different boundary conditions to the internal pipe surfaces, namely imposed heat flux, imposed temperature and convective conditions with imposed convective coefficient. The study is carried out by using a dimensionless approach and it examines the effect of the ratio between the thermophysical properties of grout and ground, the effect of the shank spacing of the internal pipes, and the effect of the fluidodynamic regime inside the pipes by means of a Biot number parameter.

The transient behaviour of the dimensionless grout thermal resistance reveals different trends for the three boundary conditions expecially for the imposed heat flux one, with a steady value higher than the others. The numerical results for convective conditions are similar to the imposed temperature ones, expecially increasing the Biot number (turbulent regime inside pipes). Moreover, the dimensionless heat capacity for unit volume plays an important role on the transient behaviour of the dimensionless grout thermal resistance, which increases as the the dimensionless heat capacity is decreased.

The steady state value of the dimensionless grout thermal resistance has been calculated in terms of either the dimensionless thermal conductivity or dimensionless shank spacing and compared with literature correlations. As expected, the resistance slightly increases with grout thermal conductivity and it increases by decreasing the shank spacing. For the single $\mathrm{U}$ pipe case, the present numerical results revealed to be in very good agreement with Bennet et al. formula when the convective boundary condition is applied at pipe inner surface.

The inaccuracy associated to calculating the grout thermal resistance with imposed temperature and then simply adding the convective thermal resistance is relevant, with an average percentage relative error with respect to the case of imposed convective boundary coefficient that increases by decreasing the Biot number.

\section{NOMENCLATURE}

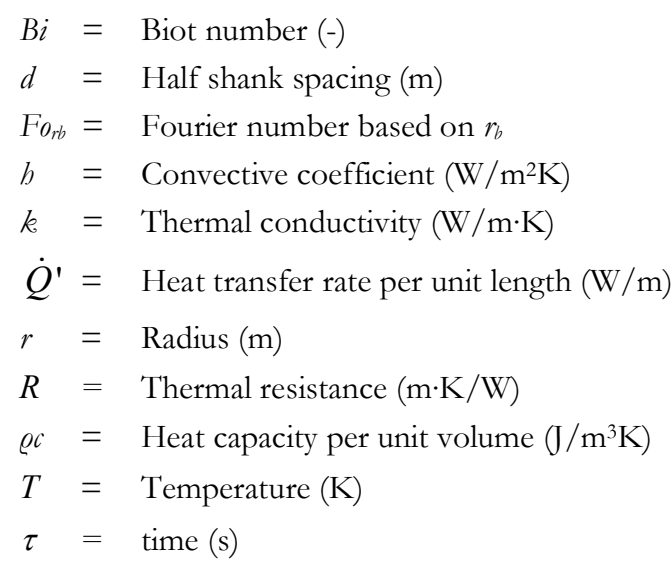

\section{Subscripts}

$$
\begin{aligned}
b & =\text { borehole } \\
c o n v & =\text { convective } \\
f & =\text { fluid } \\
g & =\text { ground } \\
g r, \infty & =\text { undisturbed ground } \\
p & =\text { pipe }
\end{aligned}
$$




\section{REFERENCES}

Bennet, J., J. Claesson and G. Hellstrom. 1987. Multipole method to compute the conductive heat transfer to and between pipes in a composite cylinder. Notes on Heat Transfer 3-1987. Dep. of Building Physics, Lund University, Sweden.

Fossa, M. and D. Dalla Pietà. 2011. Numerical evaluation of BHE thermal resistance for ground coupled heat pump applications. Alternative Source/Sinks of Heat Pump and Air-Conditioning Conference, 5-7 April, Padova, Italy.

Hellstrom, G. 1991. Ground heat storage; thermal analysis of duct storage systems. PhD Thesis, Dep. of Mathematical Physics, Lund University, Sweden.

Javed, S. and J.D. Spitler. 2016. Calculation of borehole thermal resistance. Woodhead Publishing Series in Energy, 63-95.

Lamarche, L., S. Kajl and B. Beauchamp. 2010. A review of methods to evaluate borehole thermal resistances in geothermal heat-pump systems. Geothermics 39: 187-200.

Marcotte, D. and P. Pasquier. 2008. On the estimation of thermal resistance in borehole thermal conductivity test. Renewable Energy 33: 2407-2415.

Paul, N.D. 1996. The effect of grout thermal conductivity on vertical geothermal heat exchanger design and performance. Master of Science Thesis, South Dakota State University, USA.

Priarone, A. and S. Lazzari. 2014. Short-term behavior and steady-state value of BHE thermal resistance. Proc. of European Comsol Conference 2014, Cambridge, UK.

Sharqawy, M.H., E.M. Mokheimer and H.M. Badr. 2009. Effective pipe-to-borehole thermal resistance for vertical ground heat exchangers. Geothermics 38: 271-277.

Yavuzturk, C. and J.D. Spitler. 1999. A short time step response factor model for vertical ground loop heat exchangers. ASHRAE Transactions 105: 475-485.

Zanchini, E. and S. Lazzari. 2014. New g-functions for the hourly simulation of double U-tube borehole heat exchanger fields. Energy 70: 444-455.

Zeng, H., N. Diao and Z. Fang. 2003. Heat transfer analysis of boreholes in vertical ground heat exchangers. J. Heat and Mass Transfer 46: 4467-4481. 